\title{
Determination of Surface Erosion Degree inside Dielectric Void Subjected to Partial Discharges
}

\author{
M. FLORKOWSKI* \\ ABB Corporate Research, Starowiślna 13A, 30-038 Kraków, Poland \\ (Received March 6, 2018; in final form July 26, 2018)
}

\begin{abstract}
Novel approach to determine degree of surface erosion inside void subjected to partial discharges is presented. The deterioration of the void wall is associated with a time decay of accumulated charges, which reflects changing surface conductivity. The introduced methodology is based on non-continuous, chopped partial discharges sequencing. It can allow to estimate post discharge charge decay in the void, calculated as a time constant of the decay of internal accumulated electric field from deposited charges. The chopped sequence consists of multiple series of packets containing the base waveforms, followed by the defined delay time between subsequent packets. Train of base waveforms is applied to a dielectric material and as a whole epoch repeated within given measurement time. Main focus is on the internal mechanism inside voids, especially with respect to void surface conditions and charge decay phenomena. The experiments were carried out in a gaseous voids in polyethylene filled with air at atmospheric pressure. Presented method reveals impact of deposited charges creating an internal field, which interplays with superimposed external electric field in terms of the discharge inception conditions. It was demonstrated that this methodology can reveal quantitatively charge dynamics based on partial discharges phase-resolved pattern and applied analytics. Time constant of deposited charge decay in void was estimated based on two measurements with different fill factors.
\end{abstract}

DOI: $10.12693 /$ APhysPolA.134.529

PACS/topics: 51.50.+v, 52.80.-s, 77.22.-d, 77.22.Jp

\section{Introduction}

Partial discharges (PD) are one of the most decisive factors determining condition of dielectrics exposed to high electric field stresses. Partial discharges may occur inside voids in solid dielectrics. The PD mechanism strictly depends on the type and interface between local insulation media in discharge sources. The partial discharge mechanism comprises a large variety of physical phenomena, ranging from charge carrier emission from surfaces, over leakage currents along insulator surfaces, glow discharges, charge carrier injection into liquids and solids, over phenomena of medium intensity such as electric treeing and streamers, to intense discharge types such as sparks or dry-band arcs.

For certain materials, e.g. polyethylene, the lack of gaseous inclusions does not hinder the development of discharges; they may generate in the course of the chemical decomposition of the dielectric in the electric field, and failures in the physical structure. At the stage of macroscopic changes of structure, surface and point erosion have the principal significance in the complex processes of dielectrics degradation under the influence of partial discharges. They directly or through treeing mechanisms lead to a disruptive discharge and insulation breakdown.

The processes in the dielectrics may take place under the influence of discharges triggered by two characteristic

*e-mail: marek.florkowski@pl.abb.com sources: in gaseous inclusion and in the presence of the micro-blade conducting particles in the solid material. A necessary but not sufficient condition for the occurrence of a PD is an initiatory "first" electron to start the first avalanche of the ionization process. The supply of such first electrons controls the statistical characteristics of the PD activity such as inception delay, frequency of occurrence and distribution with respect to the phase of the applied AC voltage. The mechanism of first electron generation is usually effect of volume or surface processes like radiation/ionization or emission, respectively. The $\mathrm{PD}$ inception mechanism in voids, which is one of the most common type of defects, is explained by the streamer process. The streamer discharge is a self-channelling ionization phenomenon propagating through the gas driven by a space charge distorted electric field and controlled by the interplay of ionization and electron attachment. Partial discharge exposure in gaseous voids within solid dielectrics leads to changes in surface conditions, such as roughness or morphology, as well as in physical properties, such as charge accumulation and transport, surface and bulk conductivity. Surface conductivity influences the decay of deposited charges and hence the long term inception conditions in a void [1-13].

This paper discussed novel methodology of noncontinuous partial discharge sequencing, which offers additional insight into the dynamics of PD mechanisms, in the discharge source. The multiple series of packets consisting of the base waveforms, followed by the defined delay time between subsequent packets is applied to a dielectric material and as a whole epoch repeated within given measurement time. The quantitative estimation of the decay time constant of internally accu- 
mulated charges in void is based on the two subsequent measurement with different delay time. Then the time contestant evolution in time reflects the degree of surface erosion inside the void. Non-continuous approach extends the understanding of the processes associated with partial discharge inception, propagation, neutralization, especially in context to subsequent discharges and surface processes.

\section{Discharge process in void}

Void is a gaseous inclusion in solid dielectric material and can differ from one another in terms of shape, location relative to the electrodes as well as in terms of internal structure. Furthermore, it can contain different gases at certain pressures. One void may be formed just inside dielectric material whereas others can be embedded in such a way that the metallic electrode forms one or both walls. Voids wall subjected to strong erosion can induce a treeing development having a different origin such as water, electrical or metallic due to electrode atomic migration into insulating material [14-16].

A graphical representation of a void is shown in Fig. 1. If certain conditions are fulfilled, the internal discharge will be triggered representing either the Townsend type where the discharge sequence of electron avalanches will occur and ions produce secondary electrons by surface impact. The duration of this discharge will be of the order of the ion gap crossing time. In the case of streamer discharge, the space charge field and photoionization drives propagation and forms a channel structure. The duration of the streamer discharge for is in the range of $10^{-9} \mathrm{~s}$, and thus is much shorter than the duration of the Townsend discharge, which is in the microsecond range. The electric field inside the void $E_{v}$ is a superposition of applied external field $E_{0}$ (corresponding to the voltage), transposed according to the field distribution and the shape of the void by the factor $f$ and the internal field $E_{q}$ created by the charges deposited on the void surface during a previous event

$$
E_{v}=f E_{0}+E_{q}\left(\tau_{q}\right),
$$

where $\tau_{q}$ represents the cumulative decay time constant of the $E_{q}$ field in the void.

After a discharge has taken place in the void, charges are deposited on the surface. The deposited charges contribute to the induced internal field $E_{q}$ and may also be a source where the electron generation process may start during the next discharge. However whether the induced internal field will be available depends on the decay time $\tau_{q}$ which may have different origin such as ionic drift, conduction along the void surface and recombination, or trapping into dielectric or bulk conduction [17-21]. Depending on the trapping level some electrons may be detrapped in this process for a subsequent discharge. This process will lead additionally to the time-dependent wall charge field [5]. The surface discharge time constant $\tau_{s}$ in a void is proportional to surface conduction, hence the

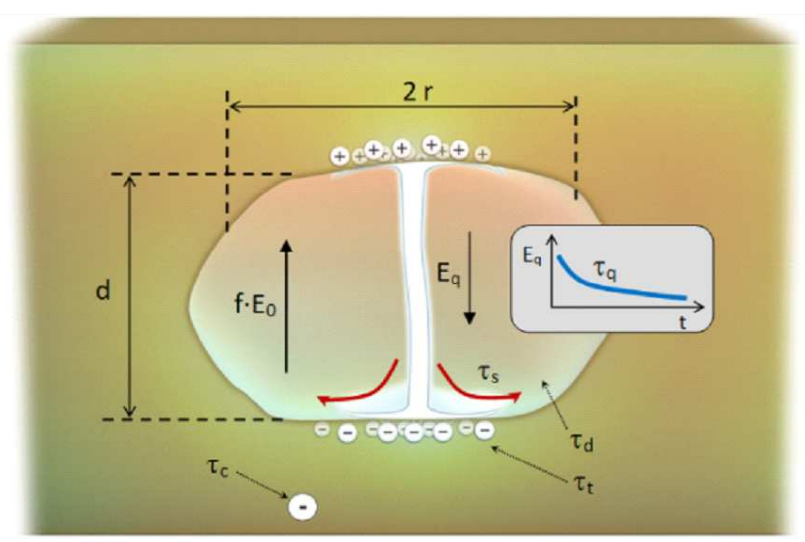

Fig. 1. Representation of the void and decay time $\tau_{q}$ of the field $E_{q}$ due to different origin such as ionic drift $\tau_{d}$, conduction along the void surface $\tau_{s}$ and recombination, trapping into dielectric $\tau_{t}$ or bulk conduction $\tau_{c} . r-$ radius of the void, $d$ - void height, $f E_{0}$ - background electric field in the void.

degree of surface erosion. It is a key component of the $E_{q}$ field decay.

In this context, the charge memory effect may be related to the ability to create and sustain of the $E_{q}$ field. The exposure of the void walls to discharges leads to surface erosion and degradation $[1,7,13,17,19]$. It was shown by [3] that the leakage current in the void in polyethylene was negligibly small in untreated specimens when compared with ones subjected to discharges. Surface conductivity of polymeric voids that have not been exposed to $\mathrm{PD}$ is typically in the range $10^{-14}$ to $10^{-16} \mathrm{~S}[3,10,18]$. When affected by PD degradation, this value may drop even by a few orders of magnitude [17]. A separate topic is related to the effective discharge area, as in bigger voids. Different areas are divided into clusters with different resistances by mapping discharge spots in selected ranges of surface resistivity occurring due to the energetic impact of PD. This effect justifies the occurrence of effective discharge area in the discharge source, as the semiconducting surface leads to the formation of cross channels in the bulk dielectric that might lead, through intermediate stages, to breakdown. This effect is impacting additionally the stochastic PD pattern modulation and being a possible origin for a formation of cross channels in the bulk insulation that can originate and lead in the long term to breakdown.

The surface charge field $E_{q}$ can be expressed as a decaying function of time

$$
E_{q}(t)=E_{q_{0}} \exp \left(\frac{-t}{\tau_{q}}\right),
$$

where $E_{q_{0}}$ is the initial charge value. $\tau_{q}$ contains the cumulative charge $q$ decay due to surface recombination, deeper trapping and bulk conduction, neutralization and other drifts.

PD event and avalanche processes impact the charge build up in a void. Directly, after the discharge occurs in- 
side the void, both surface and bulk charge neutralization and conduction processes are started, resulting in a $E_{q}$ related charge decay. In the case of very slow processes the introduced non-continuous sequence with a time delay should have theoretically no effect, unless the duration of the delay time is comparable with time constant of the charge decay. Graphically processes occurring inside void are depicted in Fig. 2. The $E_{q}$ accumulated field, represented by a green curve, follows a decay process at point A - when last discharge in the sequence before delay time $t_{d}$ occurred. With dashed lines processes for high and low surface conductivity are marked. The long term exposure of the void walls to discharges leads to surface degradation and broadening of so-called effective discharge area in bigger voids $[7,13,20,21]$. This effect is impacting additionally the stochastic PD pattern modulation and being a possible origin of bulk dielectric penetration.

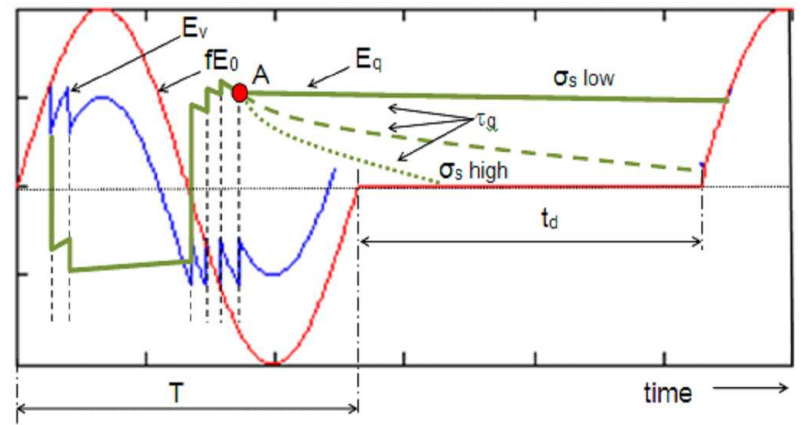

Fig. 2. Graphical illustration of the processes inside void in a non-continuous sequence. After last discharge in negative half period a decay of $E_{q}$ field is observed. At point $\mathrm{A}$ - last discharge in the sequence before delay time $t_{d}$ - the $E_{q}$ field starts to decay. $E_{v}$ - field in void, $f E_{0}$ - background field. With dashed lines processes for high and low surface conductivity are marked.

The introduced, novel methodology based on noncontinuous, chopped PD sequencing can allow to estimate post discharge charge decay in the void, calculated as a time constant $\tau_{q}$ of the decay of internal electric field $E_{q}[20]$. The method based on twofold process is illustrated in Fig. 3 (plot is not to scale, neither in magnitude nor time). Two subsequent measurements are performed for two different time delays introduced to the chopped sequence, denoted as $t_{d f f 1}$ and $t_{d} f_{f 2}$. After the last discharge in the period at negative half period, the electric field $E_{q}$ starts to decay. The decreased field $E_{q}$ results in certain deficiency comparing to continuous sequence, which should be compensated for example by higher $f E_{0}$ field, manifested by inception phase shift $\left(\phi_{i \text { cont }}\right.$ towards $\phi_{i f f 1}$ and $\left.\phi_{i f f 2}\right)$ and observed in phase resolved images.

This field drop in the void is marked by $\Delta E_{v f f 1}$ and $\Delta E_{v f f 2}$, respectively, as illustrated in Fig. 3 at points $\mathrm{A}$ and $\mathrm{B}$. The difference of electric field with respect to continuous sequence will equal to

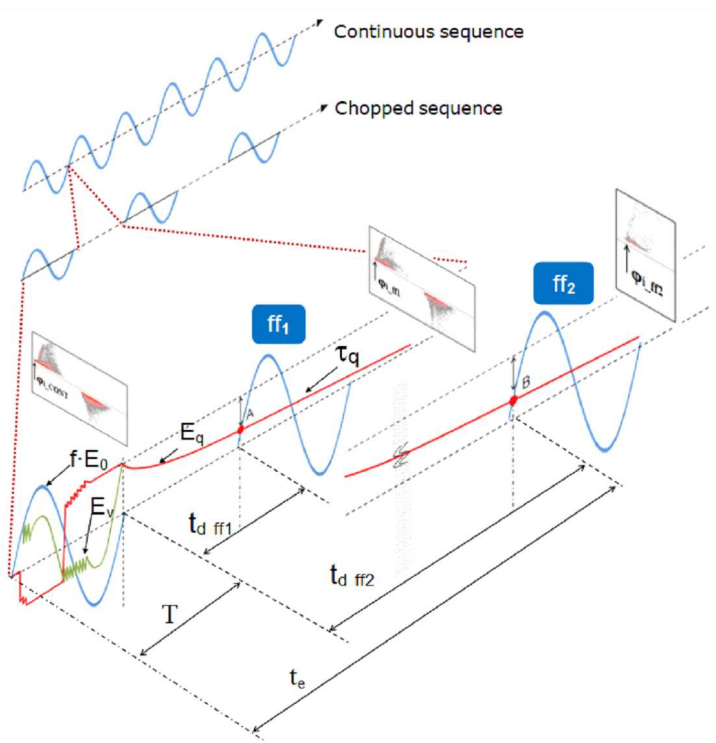

Fig. 3. Estimation of charge decay process in a noncontinuous partial discharge sequence, based on two runs with different time delays $t_{d} f_{f 1}$ and $t_{d} f f f 2_{2} . T-$ period of base waveform, $t_{e}$ - duration of the epoch.

$\Delta E_{v}=E_{v_{c} \text { ont }}-E_{v_{-} f f_{n}}$.

Introducing now the time dependence, for the moment of time $t_{f f_{n}}$ resulting from the chopped sequence with the $n$-th fill factor, one obtains

$$
\Delta E_{v}=E_{q_{0}}-E_{q_{0}} \mathrm{e}^{-t_{f_{n}} / \tau_{q}}=E_{q_{0}}\left(1-\mathrm{e}^{-t_{f_{n}} / \tau_{q}}\right) .
$$

According to the calculations presented in [22], the general solution for decay time $\tau_{q}$ can be obtained numerically from equation

$$
\frac{\left(1-\mathrm{e}^{-t_{f f_{1}} / \tau_{q}}\right)}{\left(1-\mathrm{e}^{-t_{f f_{2}} / \tau_{q}}\right)}=\frac{\sin \left(\varphi_{i_{f f_{1}}}\right)-\sin ^{2}\left(\varphi_{i_{-}} \text {cont }\right)}{\sin \left(\varphi_{i_{f f_{2}}}\right)-\sin ^{2}\left(\varphi_{i_{-}} \text {cont }\right)},
$$
assuming known parameters $t_{f f_{1}}, t_{f f_{2}}, \varphi_{i_{f f_{1}}}, \varphi_{i_{f_{f_{2}}}}$, $\varphi_{i}$ cont

\section{Experimental setup, specimen and chopped methodology}

The discussed methodology is generic, hence may be applied to various classes and forms of discharges. In this paper example related to gaseous voids in polyethylene (XLPE - cross-linked polyethylene) is presented. A specimen contained a void filled with air at atmospheric pressure. The punched void had a diameter $10 \mathrm{~mm}$, thickness $0.24 \mathrm{~mm}$. The void was clamped then between two glass plates, each $2 \mathrm{~mm}$ thick. On both sides plane stainless steel electrodes were used. The electrodes were $40 \mathrm{~mm}$ in diameter with an end curvature of $8 \mathrm{~mm}$ radius. Specimen was placed in an oil tank during measurements, performed at room temperature.

As visualised in Fig. 3, the non-continuous method is delaying consecutive base packets, introducing delay time $t_{d}$. In this way certain dynamic processes associated with 
a discharge and post discharge activities may be zoomedin. The obtained chopped PD sequence consists of a base waveform, having period $T$ and forming epoch extending through time $t_{e}$. A delayed part of the sequence is freezing somehow the regular discharge development and provides voltageless observation. In order to introduce common nomenclature with respect to the delay time, the sequence fill factor $f f$ is defined as a ratio of active exposition time $T$ (in general case the base packet can consist of several $n$ periods of base waveform), to a duration of the whole epoch, assuming the delay time as a multiple $k$ of the base waveform

$$
f f=n /(n+k) \text {. }
$$

The base waveform can assume various wave shapes. The synchronization, usually occurs at zero crossing and may start from positive or negative polarity first. The measurements were carried out in the wideband phaseresolved partial discharge acquisition (PRPDA) system. The non-continuous voltage pattern was defined in waveform generator driving the Trek (Model 20/20B) amplifier. The base waveform had period equal to $20 \mathrm{~ms}$ and was programmed in the waveform generator (Tektronix AFG 3102). The synchronization, obtained from voltage divider, was set at zero crossing of a test voltage. The $\mathrm{PD} D(\phi, q, n)$ pattern was accumulated in a $256 \times 256$ matrix in ICMsystem connected to a host computer via GPIB bus, during a $60 \mathrm{~s}$ measurement period. PD detection was obtained from coupling impedance, connected in series with a coupling capacitor $C_{k}=1100 \mathrm{pF}$ followed by a filter and preamplifier [20].

\section{Results and discussion}

In this section results obtained applying novel noncontinuous partial discharge sequencing to determine degree of surface erosion inside dielectric void are presented. The deterioration of the void wall is associated with a time decay of accumulated charges, which reflects changing surface conductivity. Applying the methodology presented in Sect. 2, the calculation can be based on two measurement with different fill factors. The measurements were performed on the void consisting of polyethylene walls, at voltage level $1.2 U_{0}$ equal to $6 \mathrm{kV}$, where $U_{0}$ corresponds to the partial discharge inception voltage at continuous sequence. In Fig. 4 there are presented PD images obtained for continuous sinusoidal run as well as for chopped sequence for polyethylene specimen. The $\mathrm{PD}$ pattern obtained at continuous sinusoidal voltage is shown in Fig. 4a and for fill factors 1:2 and 1:128 in Fig. $4 \mathrm{~b}$ and c, respectively.

Due to internal $E_{q}$ field decay the non-continuous sequence has shifted inception phase angle, whereas the negative inception phase angle is very stable. The positive PD inception phase angle is marked as $\phi_{i}$ cont for the continuous case and as $\phi_{i} C P D$ in the chopped mode. The comparison of PD inception phase angles between continuous and chopped sequence is used as an indicator for calculation of internal charge time decay in the void.

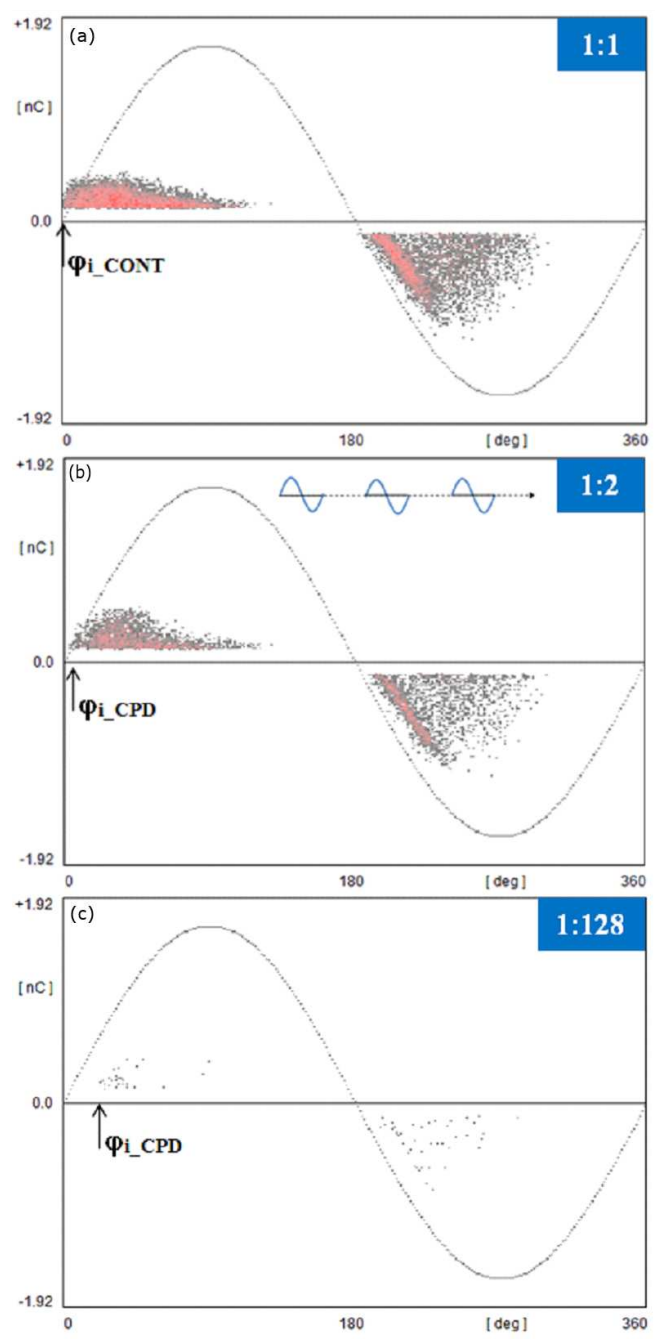

Fig. 4. PD pattern obtained in void with polyethylene wall at (a) sinusoidal voltage $6 \mathrm{kV}$ in continuous mode and (b) chopped mode $f f=1: 2\left(t_{d}=20 \mathrm{~ms}\right)$ and (c) $f f=1: 128\left(t_{d}=2540 \mathrm{~ms}\right)$.

The inception phase shift difference for polyethylene void walls, between continuous and delayed sequence by $2540 \mathrm{~ms}$ is equal to 21 degrees. The calculation based on Eq. (5), solved numerically, yields a charge decay time for polyethylene void $407 \mathrm{~ms}$. This time constant represents all processes associated with charge neutralization and conduction.

The ageing was performed on the polyethylene specimen during $70 \mathrm{~h}$ at $9 \mathrm{kV}$. The measured PD patterns along with charge distributions are shown in Fig. 5. The chopped measurement obtained with fill factor 1:2 (time delay $20 \mathrm{~ms}$ ) is presented in Fig. 5a and fill factor 1:16 (time delay $300 \mathrm{~ms}$ ) in Fig. 5b. The deterioration of void surface results in a shift of maximum charge cluster for positive half period (visible in Fig. $5 d$ ) to 480 pC at phase angle $65^{\circ}$. Performing calculations, the value of the time constant of accumulated charges after ageing is estimated at $30 \mathrm{~ms}$. It shows order of magnitude reduction comparing to non PD exposed surface. 

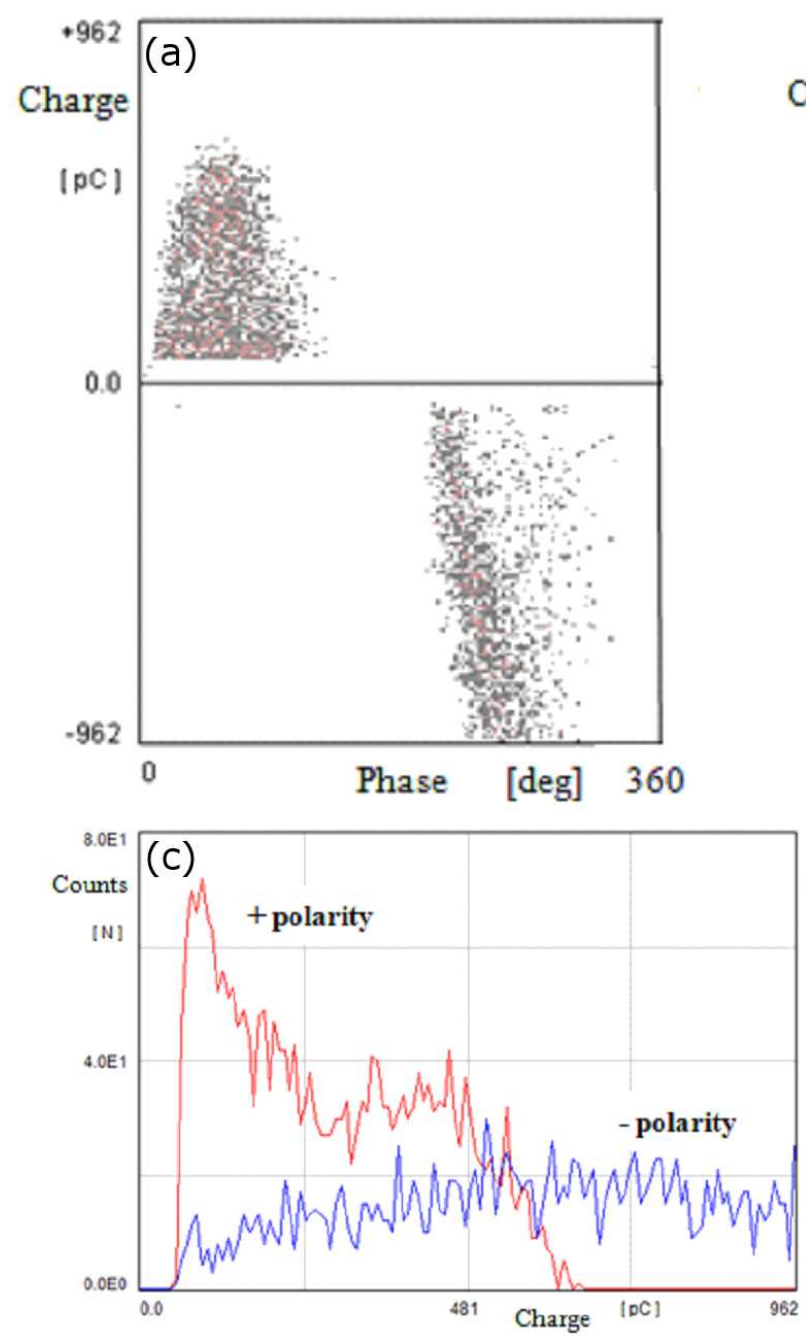
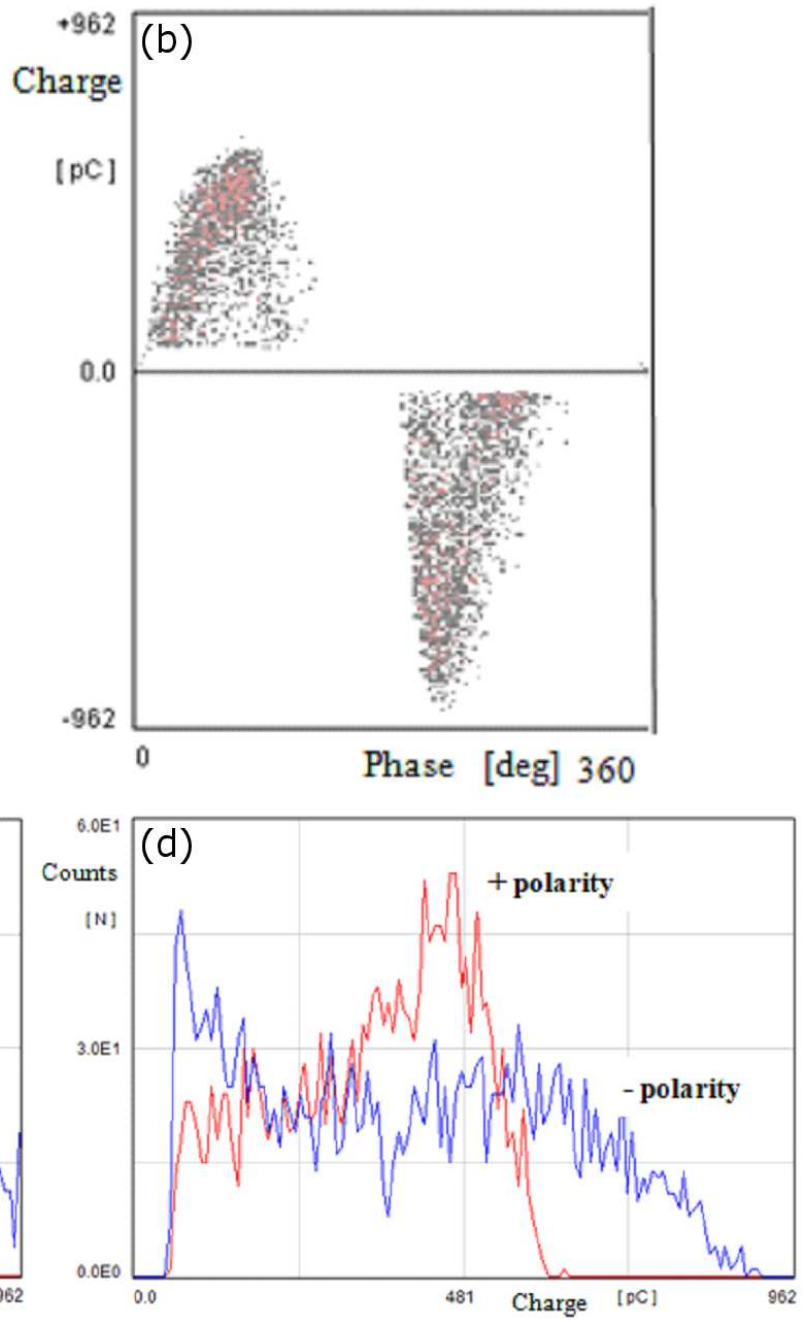

Fig. 5. PD pattern obtained for polyethylene void after aging at (a) chopped mode $f f=1: 2\left(t_{d}=20 \mathrm{~ms}\right)$ and (b) $f f=1: 16\left(t_{d}=300 \mathrm{~ms}\right)$ along with corresponding charge distributions (c) chopped $f f=1: 2$ and (d) chopped $f f=1: 16$.

The void wall surface resistivity influences the transport of charges, i.e. the higher resistivity fosters the preservation of charge reservoir and simultaneously contributes to the time lag, whereas the low resistivity tends to reduce the $E_{q}$ field. In this sense the surface charge decay through conduction causes the electron generation rate, contributing to discharge inception, to decrease much faster, increasing the statistical time lag. In addition effective discharge area inside the void modulates the charge transport through the channel.

\section{Conclusion}

This paper presents a novel approach to determine surface erosion degree inside dielectric void subjected to partial discharges. The proposed non-continuous partial discharge sequencing, introduces time delay in regular sequence providing inside to the internal mechanism inside the voids, especially related to void surface conditions and the charge decay phenomena. The measurements were performed on an embedded void in polyethylene. It was demonstrated that proposed methodology can reveal quantitatively charge dynamics based on PD phaseresolved patterns. The calculation of charge time constant was based on two consecutive measurements with two different fill factors. The associated electric field decay caused by accumulated charges reflects the deterioration and erosion degree being proportional to the surface conductivity. The performed long term ageing of the polyethylene void resulted in the surface erosion and was successfully determined. The calculated decay time was order of magnitude reduced, comparing to PD unexposed surface.

\section{References}

[1] L.A. Dissado, J.C. Fothergill, Electrical Degradation and Breakdown in Polymers, Institution of Engineering and Technology, 2008.

[2] L. Niemeyer, IEEE Trans. Dielectr. Electr. Insulat. 2, 510 (1995). 
[3] T. Tanaka, Y. Ikeda, IEEE Trans. Power Appl. Syst. 90, 2692 (1971).

[4] L. Niemeyer, B. Fruth, F. Gutffleisch, in: Proc. ISH 7th Int. Sympos. High Voltage Eng., TU Dresden, Germany 1991, p. 25.

[5] I.W. McAllister, IEEE Trans. Electr. Insulat. 27, 1202 (1992)

[6] V. Khomich, M. Malashin, S. Moshkunov, E. Shershunova, Acta Phys. Pol. A 127, 1298 (2015).

[7] B. Florkowska, in: Proc. ISH 8th Int. Sympos. High Voltage Eng., Pacifico, Yokohama 1993, p. 41.

[8] F. Gutfleisch, L. Niemeyer, IEEE Trans. Dielectr. Electr. Insulat. 2, 729 (1995).

[9] T. Tanaka, M. Uchiumi, in: Proc. Conf. on Electr. Insul. and Dielectric Phenomena (CEIDP), 1999, p. 472.

[10] T. Mizutani, Y. Taniguchi, M. Ishioka, IEEE 11th Int. Sympos. Electrets (ISE), Melbourne, Australia, Ed. R.J. Fleming, 2002, p. 15.

[11] T. Boczar, P. Witkowski, S. Borucki, A. Cichoń, Acta Phys. Pol. A 128, 299 (2015).

[12] P. Fraccz, D. Zmarzły, T. Boczar, Acta Phys. Pol. A 127, 715 (2015).
[13] K. Wu, T. Ijichi, T. Kato, Y. Suzuoki, F. Komori, T. Okamoto, IEEE Trans. Dielectr. Electr. Insulat. 12 1116 (2005)

[14] M. Florkowski, B. Florkowska, A. Rybak, P. Zydron, IEEE Trans. Dielectr. Electr. Insulat. 22, 456 (2015).

[15] M.A. Douar, A. Beroual, X. Souche, IET Gen. Transm. Distr. 10, 986 (2016).

[16] P. Frącz, T. Boczar, D. Zmarzły, T. Szczyrba, Acta Phys. Pol. A 124, 413 (2013).

[17] C. Hudon, R. Bartnikas, M.R. Wertheimer, IEEE Trans. Electr. Insulat. 28, 1 (1993).

[18] S. Kumara, Y.V. Serdyuk, S.M. Gubanski, IEEE Trans. Dielectr. Electr. Insulat. 18, 1779 (2011).

[19] H. Illias, G. Chen, P. Lewin, IET Sci. Measur. Technol. 6, 52 (2012).

[20] M. Florkowski, B. Florkowska, P. Zydron, IEEE Trans. Dielectr. Electr. Insulat. 22, 3451 (2015).

[21] C. Pan, K. Wu, M.Y. Cheng, J. Tang, IEEE Trans. Dielectr. Electr. Insulat. 24, 217 (2017).

[22] M. Florkowski, B. Florkowska, R. Włodek, IEEE Trans. Dielectr. Electr. Insulat. 24, 3831, (2017). 